\title{
Evaluation of a new extraction protocol for yeast identification by mass spectrometry
}

Frédérique Gouriet $^{1,2,}$ Farida Ghiab $^{1}$, Carine Couderc ${ }^{1,2}$, Fadi Bittar $^{1}$, Hervé Tissot Dupont ${ }^{1}$, Christophe Flaudrops ${ }^{1,2}$, Jean-Paul Casalta ${ }^{1,2}$, Bissoume Sambe-Ba ${ }^{3}$, Bécaye Fall ${ }^{3}$, Didier Raoult $^{1,2^{*}}$, Florence Fenollar ${ }^{1,2}$

${ }^{1}$ Unité de Recherche sur les Maladies Infectieuses et Tropicales Emergentes, UM 63, UMR CNRS 7278, IRD 198, Inserm 1095, Aix-Marseille Université, Faculté de médecine, 13385 Marseille, France

${ }^{2}$ Pôle de Maladies Infectieuses, Hôpital de la Timone, Rue St Pierre, 13385 Marseille

France

${ }^{3}$ Hôpital Principal de Dakar, Dakar, Senegal

Address for correspondence

Unité de Recherche sur les Maladies Infectieuses et Tropicales Emergentes, UM 63, UMR CNRS 7278, IRD 198, Inserm 1095, Aix-Marseille Université, Faculté de médecine, 27 Blvd Jean Moulin, 13385 Marseille cedex 05, France, tel: (33) 4913855 17, Fax: (33) 49187 77 72, email: didier.raoult@gmail.com

Keywords: yeast, mass spectrometry, identification

Abstract word count: 98

Text word count: 2,929 


\begin{abstract}
In this paper, we evaluate a rapid and safe pretreatment procedure using glass beads for MALDITOF yeast identification in a routine clinical laboratory avoiding the use of formic acid. We created a new yeast database library using 1,186 yeasts, including 11 references strains. The database was tested using 2,131 clinical isolates allowing accurate species-level identification in $98.9 \%(2,107 / 2,131)$ of cases with a score over 1.9 and in $99 \%(2,123 / 2,131)$ of the strains at the genus level. The new protocol is a rapid, reliable and safe procedure for the accurate identification of pathogenic Candida strains and requires minimal handling.
\end{abstract}




\section{INTRODUCTION}

Epidemiology of invasive candidiasis is a persistent public health problem (14). Candida sp. are responsible for frequent and severe infectious complications in immunocompromised patients and those undergoing invasive procedures. Although Candida albicans is the most commonly identified pathogenic yeast species, other non-albicans Candida species and species from other genera have emerged as causative agents of invasive infections (14). Six species of Candida (namely C. albicans, Candida tropicalis, Candida glabrata, Candida parapsilosis and Candida krusei) are currently considered the leading etiological agents of candidiasis. However, other Candida species have also been reported sporadically as significant pathogens. These pathogens are difficult to identify by phenotypic methods and show variable susceptibility to antifungal drugs.

There are many commercial methods available to identify yeast species involved in infection (15). Using traditional methods, including culture using chromogenic media, manual and automated biochemical kits (such as AuxaColor [Bio-Rad, Marnes-la-Coquette, France], API ID32C and Vitek 2 systems [bioMérieux, Marcy L'Etoile, France]), and immunological tests depends on the number of biochemical substrates employed and the variety and the quality of the database (15). These traditional methods usually take between 48 hours and several days for the final identification of yeast. Their accuracy may depend on the yeast species and can lead to misidentification (15). As an alternative to biochemical methods, molecular approaches (8) have been developed to provide a more rapid and accurate identification of fungi. Matrix-assisted laser desorption/ionization time-of-flight mass spectrometry (MALDI-TOF MS) has also emerged as a rapid and inexpensive method for identifying microorganisms in clinical microbiology (17) laboratories and has been used successfully to identify both bacterial and 
yeast species. Bacterial identification does not require strain pretreatment compared to yeast identification using MALDI-TOF. Yeast contains thick chitinous cell walls and formic acid extraction is thus recommended. In order to optimize the workflow of microbial laboratories as well as the accuracy rate, various protocols have been tested to simplify the MALDI-TOF MS pretreatment of yeast identification. These include direct smear without the addition of formic acid, and with the addition of $25 \%$ or $100 \%$ formic acid on-plate extraction (13).

Of the available MALDI-TOF MS mechanisms, various procedures are used. Bruker (Bremen, Germany) recommends performing an in-tube formic acid extraction for yeast prior to Biotyper analysis (13), while bioMérieux recommends an on-plate extraction with $25 \%$ formic acid prior to analysis on the VITEK MS system (23). For standard clinical workflows, it would be useful to simplify the process and the turnaround time. The direct colony method has been used to identify yeasts, but resulted in low identification rates in a large proportion of cases $(2,16)$ using the MALDI-TOF MS Bruker. In contrast, other authors reported a high rate of identification using another MALDI-TOF MS system (Shimadzu Europe, Duisburg, Germany) (20). Formic acid (FA), also called methanoic acid, is a strong, colorless acid. FA is widely used in industry as a food preservative, as well as in the production of textiles and pesticides. It is well known to be hazardous to human health. FA can cause direct or indirect toxicity. Direct eye or skin contact with liquid or vapor containing FA can also lead to serious acid burns. Oral ingestion is also life threatening. Moreover, FA can lead to memory loss, confusion, seizure, coma and cardiac arrest. Finally, chronic exposure may cause kidney or liver damage and skin allergies (9). An accident took place in our lab, resulting in formic acid skin burns, so we decided to change our extraction procedure in order to promote the health and safety of our technicians. 
In this study, we evaluate a rapid and safe pretreatment procedure for MALDI-TOF yeast identification in a standard clinical laboratory avoiding the use of formic acid. We developed a formic acid free extraction protocol using glass beads and microFlex MALDI-TOF MS. We also created a new yeast database library using 1,186 yeasts including 11 references strains (from DSMZ) and tested the robustness and the accuracy of this database using an additional 2,269 clinical isolates.

\section{MATERIAL AND METHODS}

\section{Creation of our yeast database library using reference strains and clinical isolates}

In this study we constructed the yeast database library: 11 reference strains purchased from DSMZ (Braunschweig, Germany) and 1,175 strains collected retrospectively from our Microbiology Laboratory at La Timone Hospital in Marseille, France (Table1). All isolates were cultured on Sabouraud's dextrose agar (Oxoid, Dardilly, France) and incubated for 48 hours at $30^{\circ} \mathrm{C}$ to ensure purity prior to testing.

These strains were initially identified by MicroFlex mass spectrometer (Bruker Daltonics, Bremen, Germany) using the standard extraction protocol as recommended by the manufacturer.

\section{Validation of the yeast database library}

The accuracy of the created yeast library for MALDI-TOF identification was evaluated by analyzing 2,131 additional clinical isolates (Table 2), collected prospectively from our Microbiology Laboratory at La Timone Hospital in Marseille, France between October 2014 and July 2015. These were also identified, simultaneously, by MicroFlex mass spectrometer (Bruker Daltonics, Bremen, Germany) using the standard extraction protocol recommended by the manufacturer. All isolates were recovered from the routine examination of clinical specimens submitted to the microbiological laboratory, such as blood cultures, urine and respiratory tract 
samples, pus, biopsies, swab from any sites of the body, and wound specimens. All isolates were grown on bacterial media: blood agar (Becton Dickinson, Le Pont de Claix, France) or chocolate agar (Becton Dickinson, Le Pont de Claix, France). In the case of mixed culture, the yeast strain was isolated on Sabouraud's dextrose agar (Oxoid, Dardilly, France) and incubated for 48 hours at $30^{\circ} \mathrm{C}$ to ensure purity prior to testing.

For external validation purposes, we also analyzed 34 strains obtained from the Labazur Laboratory (Marignane, France) and 104 strains obtained from the Microbiology Laboratory at the main hospital in Dakar, Senegal (Table 2), using the new protocol. These strains were initially identified using mass spectrometry VITEK®MS (bioMérieux, Marcy L’Etoile, France) in the Labazur Laboratory and VITEK®MS RUO in Dakar (bioMérieux, Marcy L’Etoile, France) by applying the standard extraction protocol recommended by the manufacturer.

\section{Yeast strain preparation for MALDI-TOF mass spectrometry analyses}

Both the standard extraction protocol using formic acid and the extraction protocol using glass beads were performed simultaneously for each strain. Two or three freshly grown colonies were transferred, using a plastic inoculation loop, into a $1.5 \mathrm{ml}$ polypropylene SafeSeal microtube (Sarstedt 72.706, Mamay, France) containing $300 \mu \mathrm{L}$ of ultra-pure water. Then, 900 $\mu \mathrm{L}$ of $100 \%$ ethanol was added. The microtube was briefly vortexed and centrifuged at $13,000 \times \mathrm{g}$

for two minutes and the supernatant was discarded. Formic acid (70\%) (Sigma, Aldrich, Lyon, France) and acetonitrile (Prolabo $\mathrm{BDH})$ were added to the yeast pellet in a 1:1 (v/v) ratio (solution prepared daily). The mixture was quickly vortexed and centrifuged for two minutes at $13,000 \times g$. The supernatant $(1.5 \mu \mathrm{L})$ was spotted onto a $96-$ well stainless steel target plate (Bruker Daltonik) and air-dried. Finally, $1.5 \mu 1$ of the MALDI matrix $\alpha$-cyano-4hydroxycinnamic (prepared daily), at a final saturation concentration, in an organic solvent 
mixture of $50 \%$ acetonitrile/2.5\% trifluoroacetic acid solution, was applied onto the dried spot. Each sample was spotted four times to ensure reproducibility of spectra. An Escherichia coli protein extract test standard (DH5 alpha, Bruker Daltonik) was used to calibrate the instrument and a negative extraction control (matrix only) was included for each run.

\section{New extraction protocol using glass beads (GBP)}

Two or three freshly grown colonies were transferred, using a plastic inoculating loop, into a 2 ml polypropylene microtube containing $100 \mu \mathrm{L}$ of ultra-pure water/acetonitrile (50/50) and two small spoons of stainless steel micro spatula (reference 076188, Dutscher, Brumath, France) of glass beads were added. The mixture was then briefly vortexed and homogenized by a FastPrep 24 (FastPrep®-24 Instrument, MP BIOMEDICALS, Illkirch, France) for 20 seconds at a speed of $4 \mathrm{~m} / \mathrm{s}$. The homogenization was repeated five times. The mixture was then centrifuged for two minutes at $13,000 \times \mathrm{g}$ and the supernatant $(1.5 \mu \mathrm{L})$ was spotted onto a 96-well stainless steel MALDI target plate (Bruker Daltonik) and was air-dried. A $1.5 \mu 1$ of the MALDI matrix $\alpha$ cyano-4-hydroxycinnamic (prepared daily), in an organic solvent mixture of $50 \%$ acetonitrile $/ 2.5 \%$ trifluoroacetic acid solution was applied onto the dried spot. Each sample was spotted four times to ensure reproducibility of spectra. An Escherichia coli protein extract test standard (Bruker Daltonik) was used to calibrate the instrument and a negative extraction control (matrix only) was included for each run.

\section{Mass spectra acquisition and data analysis}

MALDI-TOF MS analysis of all strains was performed on a MicroFlex mass spectrometer (Bruker Daltonics, Bremen, Germany). The spectra were recorded in the linear positive mode at a laser frequency of $60 \mathrm{~Hz}$ within a mass range from $\mathrm{m} / \mathrm{z}$ 2,000 to 20,000. For each spectrum, 240 laser shots in 40-shot steps from different positions of the target spot were collected and 
analyzed. The spectra were externally calibrated by using Escherichia coli ribosomal proteins (Bruker Daltonics). To identify an unknown microorganism, the acquired spectrum was loaded with the MALDI Biotyper Software (Bruker Daltonics) and analyzed using the standard patternmatching algorithm, which compared the spectrum acquired with those present in the library(update_version 4 .0.0.1_4613-5627). The fingerprints of unknown samples were compared to the fingerprints for all entries in the database and the results were listed in a ranking table. The results of the pattern-matching process were expressed as log score values, which ranged from 0 to 3 . Identification scores were interpreted as follows: if the 4 spectra had a score $\geq$ than 1.9 , species identification was validated; if the score $<1.9$ and $\geq 1.7$ the genus identification was validated; finally score of below 1.70, was interpreted as "no reliable identification" $(18,19)$.

\section{Spectral analysis using the Bruker MALDI Biotyper creation data base}

FlexAnalysis Software (Bruker Daltonics) was used for visual inspection and mass spectra processing such as smoothing, normalization, baseline subtraction and peak picking. Acceptable spectra for reference strain were defined by the experimenter. The four spectra must be stackable, the baseline must be flat, sufficient intensity must be around 1.10e4. Main spectra projections (MSP) were generated considering the four spectra average obtained by the second

protocol and were saved separately from the Biotyper database (Bruker Daltonics). The name of the MSP species was given by the first protocol. The addition of a given reference strain was made using the "MSP creation" function in the MALDI Biotyper software (version 3.0) comprising Bruker's default parameters (maximum mass error of each single spectrum, 2,000; desired mass error for the MSP, 200; desired peak frequency minimum, 25\%; maximum desired peak number for the MSP, 70). 


\section{DNA extraction, PCR amplification and DNA sequencing}

In the event of discrepancy in the identification results between the two protocols or in the event of a low score (i.e. <1.7) of MALDI TOF results of any protocol used, ITS sequencing was used to give the final identification of the yeast strain as previously described (6).

\section{RESULTS}

Following the construction of our database using the new glass beads protocol and 1,186 yeast strains, we prospectively analyzed, from October 2014 to July 2015, all yeast isolates recovered from clinical specimens in our standard laboratory (La Timone Hospital, Marseille, France) using both the FA and the glass beads methods. Finally, a total of 2,131yeasts (Table 1) were isolated from various deep and superficial samples during this period. Most isolated stains (93\%) belonged to four Candida species (Table 1) including Candida albicans (78.4\%), Candida tropicalis (5.9\%), Candida glabrata (4.8\%), and Candida parapsilosis (3.9\%) (Table 1). Yeasts were most frequently isolated from urine samples $(622,29.2 \%)$, genital samples $(592,27.8 \%)$, respiratory tract samples, including bronchial aspiration, broncho-alveolar fluid and tracheal aspiration $(422,19.8 \%)$, blood culture samples $(98,4.6 \%)$, skin samples $(106,4.97 \%)$, pharyngeal samples $(66,3 \%)$, newborn gastric samples $(30,1.5 \%)$, and abscesses $(11,0.5 \%)$.

The new extraction method using the glass beads protocol enabled us to identify 2,107 $(98.9 \%)$ yeast isolates to the species level with spectral scores $\geq 1.9$ and $16(0.75 \%)$ isolates to the genus level with spectral scores ranging between 1.7 and1.9 (Table 2). Only eight isolates $(0.4 \%)$ which presented scores below 1.7 , failed to be identified using the new extraction protocol against our constructed database. By applying the standard extraction protocol, it was possible to identify 1,431 yeasts $(67.15 \%)$ to the species level with spectral scores $\geq 1.9,673$ yeasts (31.6\%) to the genus level with spectral scores between 1.7-1.9, and $27(1.3 \%)$ isolates 
without reliable identification (scores <1.7) (Table 2). The final identification of these nonidentified strains was performed using molecular techniques and the results were in keeping with those found by our proposed protocol. Thus, using the new developed extraction method, we could significantly identify more yeast isolates to the species level than the FA protocol $(\mathrm{p}<0.0005)($ Table 2$)$.

Moreover, additional yeast strains (138 isolates) from other microbiology laboratories were tested for external validation. Although major concordant results between the two protocols were obtained for the 34 clinical isolates received from the Labazur Laboratory, the glass beads method correctly identified all tested isolates with scores $\geq 2$. Meanwhile, 27 strains (79.4\%) were identified at score $\geq 1.9$ using FA extraction and the seven remaining strains (20.6\%) had scores ranging between 1.7 and 1.9. Similarly, the 104 clinical isolates obtained from the microbiology laboratory in Dakar, presented concordant identification using both methods. However, the glass beads method allowed all strains with spectral scores $\geq 2$ to be correctly identified, compared to 69 strains with scores $\geq 1.9$ and 35 strains with scores between 1.7-1.9 using the FA extraction method.

\section{DISCUSSION}

The current study evaluates a new protein extraction protocol, avoiding the use of formic acid, for the identification of yeasts cultured from clinical specimens using a Bruker Daltonics MicroFlex Lt biotyper. Firstly, we constructed an in-house database including 1,186 reference spectra. This new database was then tested using 2,131 clinical isolates resulting in accurate species-level identification in $98.9 \%(2,107 / 2,131)$ of the cases with a score $>1.9$ and in $99 \%$ $(2,123 / 2,131)$ of the strains at the genus level. Most of the species tested in this study reflected 
their occurrence in clinical specimens; C. albicans, C. glabrata, C. tropicalis, C. krusei, and C. parapsilosis represent about $95 \%$ of the yeasts tested.

Glass beads have previously been used for protein extraction before Penicillium species identification by MALDI-TOF MS. Both conidia and hyphae were collected from culture and resuspended in a mixed of acetonitrile/trifluoroacetic acid and glass beads, vortexed, and centrifuged. The supernatant was then used to perform the identification (7). El Khéchine et al. (5) used also glass beads to facilitate the complete cellular disruption of mycobacterial samples for MALDI-TOF MS identification, followed by centrifugation and FA-acetonitrile treatment. The FA extraction procedure, as recommended by the manufacturer, is laborious for laboratory workflow (21). Most alternative protocol extractions for fungi try to simplify the extraction procedures by removing the centrifugation steps. Direct smears of fungi colony with formic acid $(25 \%, 70 \%)$ overlay applied on the target plate $(10,21)$ are the most alternative process used.

Direct colony methods without FA, were adapted for yeast but were associated with no acceptable scoring spectra $(16,20)$. Cassagne et al. reported that the complete extraction pretreatment procedure has had a significant impact on the performance of MALDI-TOF MS to give a reliable identification rate (3).

In terms of safety, our results are promising as they involve a non-hazardous procedure which enabled us to identity the most common yeast species in human pathology. Moreover, the spectral scores were higher compared to the standard protocol $(\mathrm{p}<0.0005)$. Most of our yeasts were immediately identified from the first isolation on chocolate or blood agar. Similarly, Cassagne et al. reported significantly lower log scores with the Sabouraud gentamicin chloramphenicol (3) compared to the bacterial medium (chocolate or blood agar) or CHROMagar plates. Finally, our protocol saves time. The FA protocol required the suspension 
of colonies in microtubes, mixing with various solvents and several centrifugations, takes around nine minutes for one specimen, while the glass beads protocol, which includes only one centrifugation step, takes around four minutes.

Identification failures using our method were principally due to the restricted number of reference spectra in the data base. This includes eight isolates/2,131giving no reliable identification score $<1.7$ and having few or no references $($ spectra $<5)$ in the data base. When appropriate reference organisms were added to the new database, all clinical isolates were correctly identified. One advantage of MALDI-TOF MS identification is that the spectral database is easy to update, leading to a rapid improvement of the identification rate $(11,22)$. Our identification rates are slightly higher than those reported by Marklein et al. (92\%) (3), McTaggart et al. (93.1\%), Bader et al. (1) and Dhiman et al. (>90\%) (4). One explanation for this good performance could be that the strains tested in our study represent more than $90 \%$ of the five most common yeast species in human samples. However, the use of an in-house database can improve the performance of yeast identification (3). Yeast cell lysate produced by mechanical glass bead disruption is widely used in a variety of applications, including those used for the analysis of native function (protein-protein interaction or enzyme assays or membrane fractionations (12). A small fraction of yeast proteins (1-10\%) seem to be susceptible to such induced aggregation.

The results of this study clearly demonstrate the efficiency of our new extraction protocol for yeast identification by MALDI-TOF MS. The new protocol is a rapid, reliable, and safe procedure for the accurate identification of pathogenic Candida strains and requires minimal handling. As the intrinsic resistance of yeasts to antifungal agents is generally predictable according to the fungal species, it is likely that the benefit of the substantial time savings implied 
by this method, together with the increased resolution, will improve yeast diagnosis and have a profound impact on patient care.

\section{Acknowledgments}

We thank Caroline Albertelli, Geoffray Abry, Estelle Guillard, Séverine Guitton, Sébastien

Savary, Marion Salemme, and Audrey Preire for their technical assistance. We thank Violaine

Bernier for corrections to the English text. 


\section{References}

1. Bader, O., M. Weig, L. Taverne-Ghadwal, R. Lugert, U. Gross, and M. Kuhns. 2011. Improved clinical laboratory identification of human pathogenic yeasts by matrix-assisted laser desorption ionization time-of-flight mass spectrometry. Clin.Microbiol.Infect. 17:1359-1365.

2. Cassagne, C., A. L. Cella, P. Suchon, A. C. Normand, S. Ranque, and R. Piarroux. 2013. Evaluation of four pretreatment procedures for MALDI-TOF MS yeast identification in the routine clinical laboratory. Med.Mycol. 51:371-377..

3. Cassagne, C., A. C. Normand, L. Bonzon, C. L'Ollivier, M. Gautier, F. Jeddi, S. Ranque, and R. Piarroux. 2015. Routine identification and mixed species detection in 6,192 clinical yeast isolates. Med.Mycol.54 :256-65..

4. Dhiman, N., L. Hall, S. L. Wohlfiel, S. P. Buckwalter, and N. L. Wengenack. 2011. Performance and cost analysis of matrix-assisted laser desorption ionization-time of flight mass spectrometry for routine identification of yeast. J.Clin.Microbiol. 49:1614-1616.

5. El, K. A., C. Couderc, C. Flaudrops, D. Raoult, and M. Drancourt. 2011. Matrix-assisted laser desorption/ionization time-of-flight mass spectrometry identification of mycobacteria in routine clinical practice. PLoS One 6:e24720.

6. Hamad, I., M. B. Keita, M. Peeters, E. Delaporte, D. Raoult, and F. Bittar. 2014. Pathogenic eukaryotes in gut microbiota of western lowland gorillas as revealed by molecular survey. Sci.Rep. 4:6417.

7. Hettick, J. M., B. J. Green, A. D. Buskirk, M. L. Kashon, J. E. Slaven, E. Janotka, F. M. Blachere, D. Schmechel, and D. H. Beezhold. 2008. Discrimination of Penicillium isolates by matrix-assisted laser desorption/ionization time-of-flight mass spectrometry fingerprinting. Rapid.Commun.Mass.Spectrom. 22:2555-2560. 
8. Leaw, S. N., H. C. Chang, H. F. Sun, R. Barton, J. P. Bouchara, and T. C. Chang. 2006. Identification of medically important yeast species by sequence analysis of the internal transcribed spacer regions. J.Clin.Microbiol. 44:693-699.

9. Liesivuori, J. and H. Savolainen. 1991. Methanol and formic acid toxicity: biochemical mechanisms. Pharmacol.Toxicol. 69:157-163.

10. Mancini, N., C. E. De, L. Infurnari, A. Vella, N. Clementi, L. Vaccaro, A. Ruggeri, B. Posteraro, R. Burioni, M. Clementi, and M. Sanguinetti. 2013. Comparative evaluation of the Bruker Biotyper and Vitek MS matrix-assisted laser desorption ionization-time of flight (MALDI-TOF) mass spectrometry systems for identification of yeasts of medical importance. J.Clin.Microbiol. 51:2453-2457.

11. Marklein, G., M. Josten, U. Klanke, E. Muller, R. Horre, T. Maier, T. Wenzel, M. Kostrzewa, G. Bierbaum, A. Hoerauf, and H. G. Sahl. 2009. Matrix-assisted laser desorption ionization-time of flight mass spectrometry for fast and reliable identification of clinical yeast isolates. J.Clin.Microbiol. 47:2912-2917.

12. Papanayotou, I., B. Sun, A. F. Roth, and N. G. Davis. 2010. Protein aggregation induced during glass bead lysis of yeast. Yeast 27:801-816.

13. Pence, M. A., T. E. McElvania, M. A. Wallace, and C. A. Burnham. 2014. Comparison and optimization of two MALDI-TOF MS platforms for the identification of medically relevant yeast species. Eur.J.Clin.Microbiol.Infect.Dis. 33:1703-1712.

14. Pfaller, M. A. and D. J. Diekema. 2007. Epidemiology of invasive candidiasis: a persistent public health problem. Clin.Microbiol.Rev. 20:133-163.

15. Pincus, D. H., S. Orenga, and S. Chatellier. 2007. Yeast identification--past, present, and future methods. Med.Mycol. 45:97-121.

16. Pinto, A., C. Halliday, M. Zahra, H. S. van, T. Olma, K. Maszewska, J. R. Iredell, W. Meyer, and S. C. Chen. 2011. Matrix-assisted laser desorption ionization-time of flight mass spectrometry identification of yeasts is contingent on robust reference spectra. PLoS One 6:e25712. 
17. Posteraro, B., L. Efremov, E. Leoncini, R. Amore, P. Posteraro, W. Ricciardi, and M. Sanguinetti. 2015. Are the Conventional Commercial Yeast Identification Methods Still Helpful in the Era of New Clinical Microbiology Diagnostics? A Meta-Analysis of Their Accuracy. J.Clin.Microbiol. 53:2439-2450.

18. Seng, P., M. Drancourt, F. Gouriet, S. B. La Scola, P. E. Fournier, J. M. Rolain, and D. Raoult. 2009. Ongoing revolution in bacteriology: routine identification of bacteria by matrix-assisted laser desorption ionization time-of-flight mass spectrometry. Clin.Infect.Dis. 49:543-551.

19. Seng, P., J. M. Rolain, P. E. Fournier, S. B. La, M. Drancourt, and D. Raoult. 2010. MALDITOF-mass spectrometry applications in clinical microbiology. Future Microbiol. 5:1733-1754.

20. Seyfarth, F., C. Wiegand, M. Erhard, Y. Graser, P. Elsner, and U. C. Hipler. 2012. Identification of yeast isolated from dermatological patients by MALDI-TOF mass spectrometry. Mycoses. $55: 276-280$.

21. Van Herendael, B. H., P. Bruynseels, M. Bensaid, T. Boekhout, B. T. De, I. Surmont, and A. H. Mertens. 2012. Validation of a modified algorithm for the identification of yeast isolates using matrix-assisted laser desorption/ionisation time-of-flight mass spectrometry (MALDI-TOF MS). Eur.J.Clin.Microbiol.Infect.Dis. 31:841-848.

22. Van Veen, S. Q., E. C. Claas, and E. J. Kuijper. 2010. High-throughput identification of bacteria and yeast by matrix-assisted laser desorption ionization-time of flight mass spectrometry in conventional medical microbiology laboratories. J.Clin.Microbiol. 48:900-907.

23. Westblade, L. F., R. Jennemann, J. A. Branda, M. Bythrow, M. J. Ferraro, O. B. Garner, C. C. Ginocchio, M. A. Lewinski, R. Manji, A. B. Mochon, G. W. Procop, S. S. Richter, J. A. Rychert, L. Sercia, and C. A. Burnham. 2013. Multicenter study evaluating the Vitek MS system for identification of medically important yeasts. J.Clin.Microbiol. 51:2267-2272. 


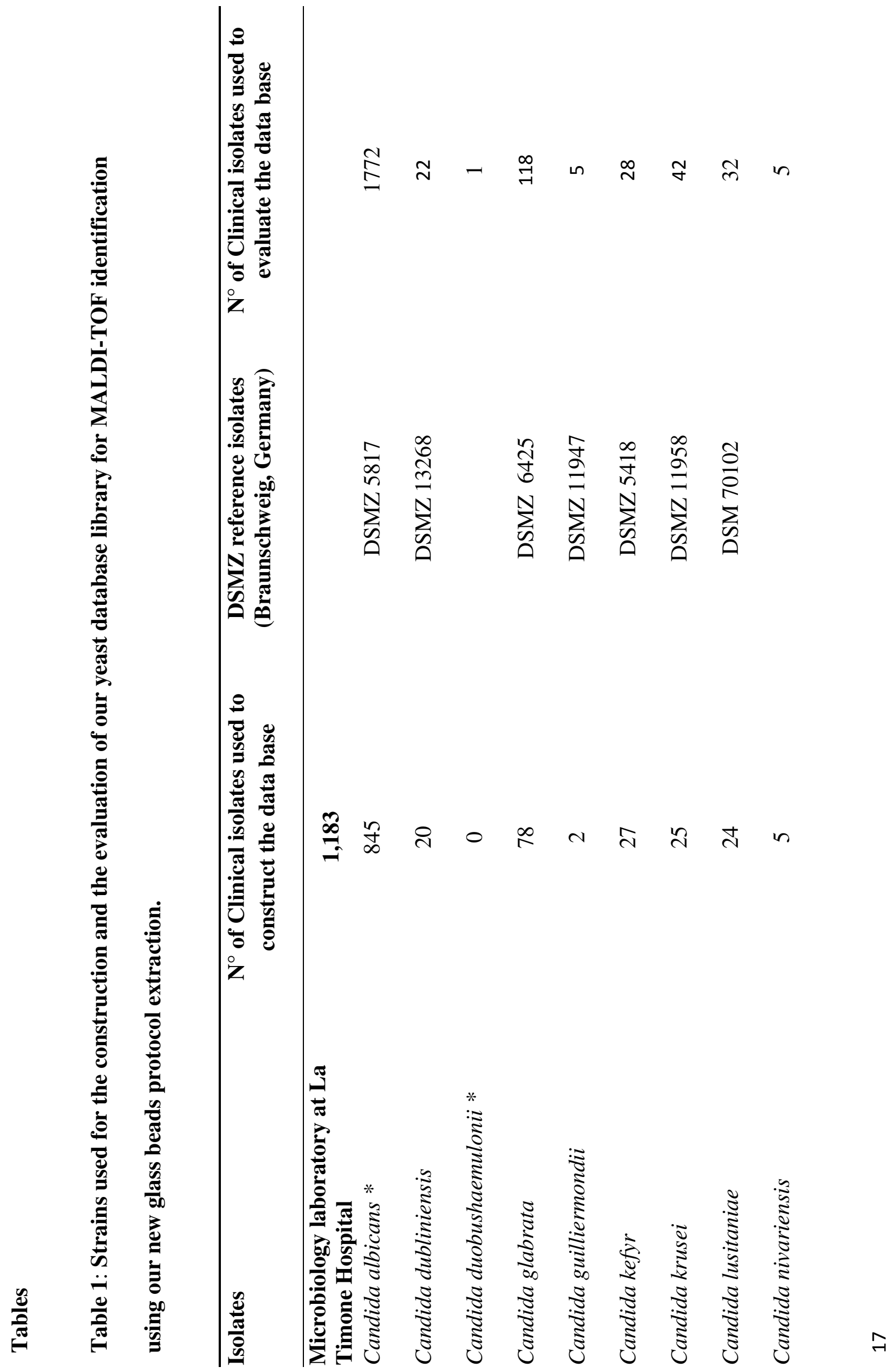




$$
\begin{aligned}
& -\wedge \underset{+}{\stackrel{+}{\triangleleft}}-\sim \sim m \\
& \stackrel{n}{2} \quad \stackrel{n}{2}
\end{aligned}
$$

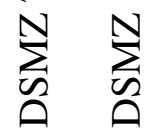

$$
\begin{aligned}
& \stackrel{m}{m} \frac{N}{n} \\
& \text { 施 } \sum^{N} \\
& \text { - m } \sigma \infty 0 N-6-0 \mathrm{~N}
\end{aligned}
$$

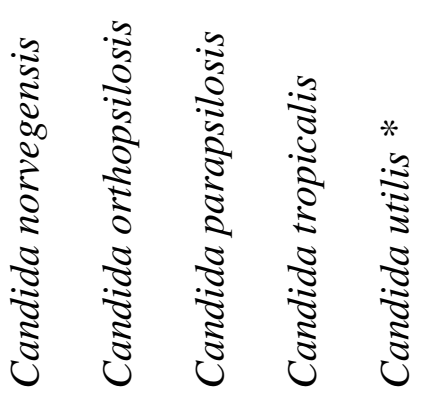

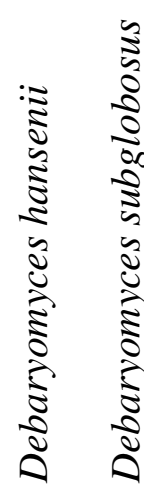

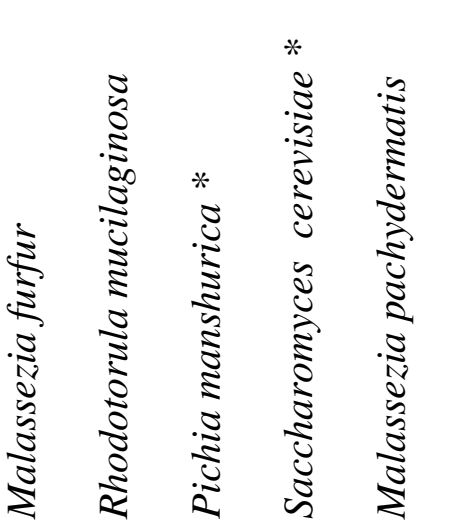

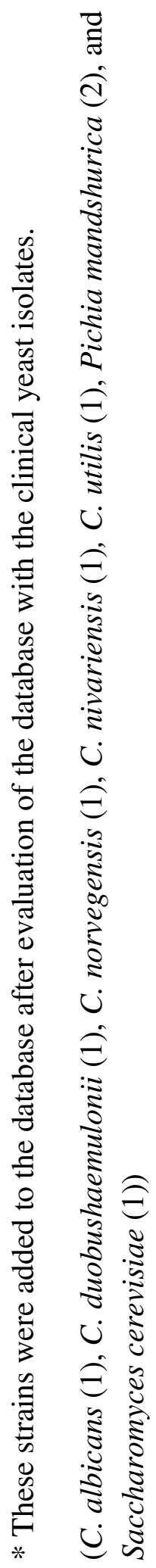



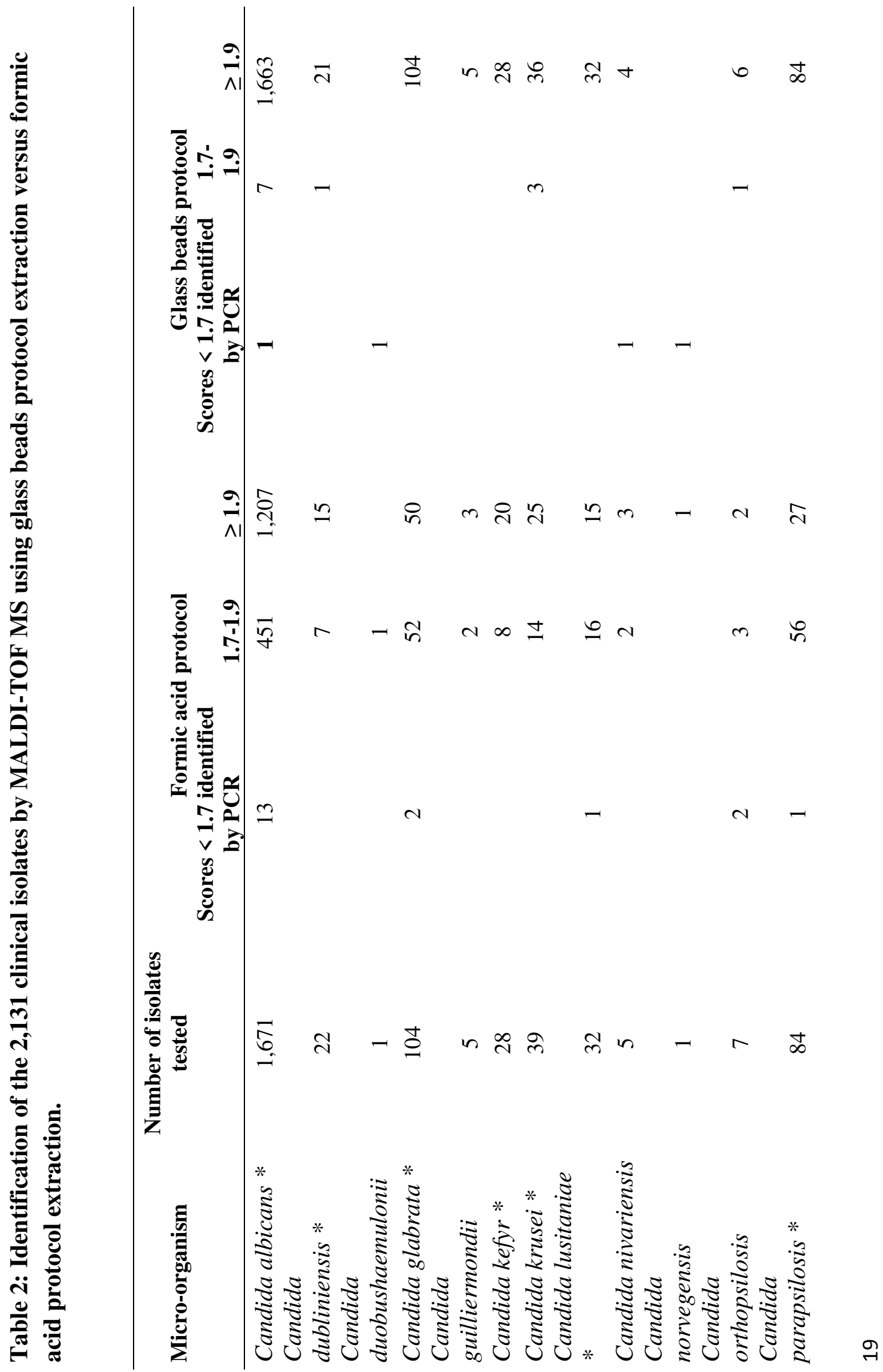


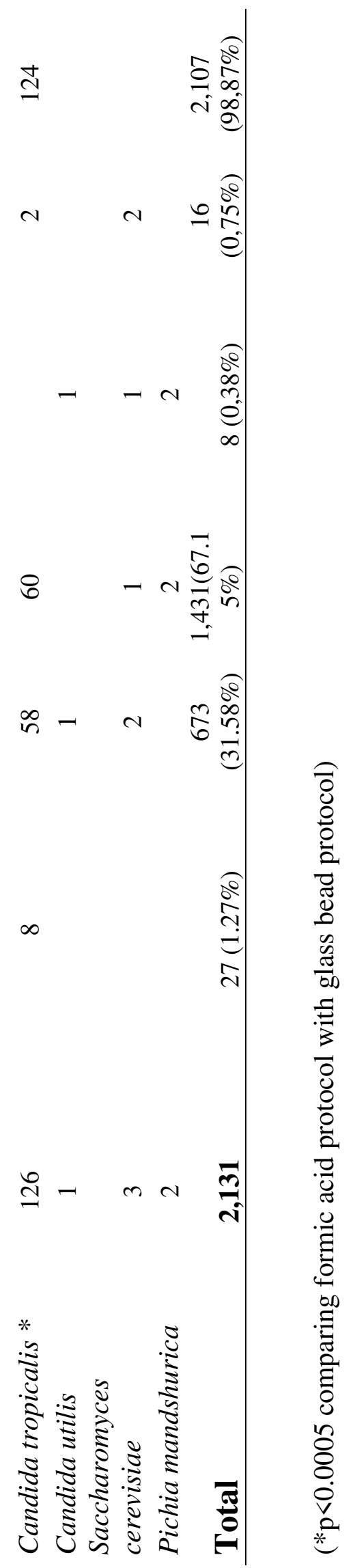

